\title{
Algorithms for Sensor Networks: What Is It Good for?
}

\author{
Roger Wattenhofer \\ Distributed Computing Group \\ Computer Engineering and Networks Laboratory \\ Information Technology and Electrical Engineering \\ ETH Zurich \\ Switzerland \\ wattenhofer@tik.ee.ethz.ch
}

\begin{abstract}
Absolutely nothing!? The merit of theory and algorithms in the context of wireless sensor and ad hoc networks is often questioned. Admittedly, coming up with theory success stories that will be accepted by practitioners is not easy. In my talk I will discuss the current score of the Theory vs. Practice game, after playing seven years for the Theory team. Probably due to a "seven year itch", I recently also started playing for the Practice team.
\end{abstract}

